\title{
The Reynolds Equation Method for Crankshaft's oil Film Stiffness
}

\author{
Gao Lei ${ }^{1, a}$, Zhang Hongxin ${ }^{1, b^{*}}$, Xu Wei ${ }^{1, c}$, Shu Pei $i^{1, d}$ \\ ${ }^{1} 308$ Ningxia Road, Qingdao, Shandong, P.R.C, 266071 \\ agaoleiqdu@163.com, bqduzhx@126.com, '1107121745@qq.com , dqdushupei1988@126.com
}

\begin{abstract}
Keywords: oil film stiffness; crankshaft; Reynolds equation; MATLAB
Abstract. The paper give a method according to the formation mechanism of the crankshaft's oil film lubrication, positioning Newtonian fluid lubrication, determine the boundary conditions of the Reynolds equation. Then calculate the oil film thickness equations according to its mechanical principle, simplify out the Reynolds equation and the dimensionless simplified under the condition. Using successive over relaxation (SOR) method in MATLAB programming obtain the oil film stiffness of the Hydraulic Confined Piston Engine's (HCPE) crankshaft.
\end{abstract}

\section{Introduction}

In the optimization analysis of the crankshaft, we usually have to consider the influence of oil film. In the study of the oil film stiffness, it can be based on the dynamic journal bearing. The traditional methods to solve the oil film stiffness of the crankshaft are simplified the Reynolds equation into one dimensional of bearing that infinite wide or narrow, they just reflect the oil film pressure distribution in the circumferential or in the radial direction, the calculated parameters do not conform to the actual situation. In recent years, along with the improvement of calculation method and the development of computer software and hardware technology, it is possible to calculate the related parameters of finite length bearing. This paper based on Reynolds equation and finite difference method, with the aid of MATLAB software to solve the oil film stiffness of the Hydraulic Confined Piston Engine's crankshaft.

\section{Reynolds equation derivation and its dimensionless simplified}

Reynolds equation derivation and its physical interpretation. The main steps of Reynolds equation derivation: obtain the fluid's velocity distribution along the direction of oil film thickness according to the condition of the micro-unit force balance. Integrate velocity along the direction of oil film thickness to get the flow, with flow continuity conditions, the final common forms of Reynolds equation is deduced.

$$
\frac{\partial}{\partial x}\left(\frac{\rho h^{3}}{\eta} g \frac{\partial p}{\partial x}\right)+\frac{\partial}{\partial y}\left(\frac{\rho h^{3}}{\eta} g \frac{\partial p}{\partial y}\right)=6\left[\frac{\partial}{\partial x}(U \rho h)+\frac{\partial}{\partial y}(V \rho h)+2 \rho \frac{\partial h}{\partial t}\right]
$$

In the coordinate axes, $\mathrm{x}$ is the tangential direction, $\mathrm{y}$ is the radial direction, $V$ is the axial velocity, $U$ is the tangential velocity, $p$ is the pressure, $\mathrm{t}$ is the time, $\rho$ is the density of the oil, $h$ is the oil film's thickness. The left of Reynolds equation means lubrication oil film pressure change at the lubrication surface along the $\mathrm{x}$, $\mathrm{y}$ position.

Hydrodynamic effect and squeeze effect are the main factors to cause the lubrication oil film pressure.

Oil film thickness equations. This is the figure to solve the oil film thickness: 


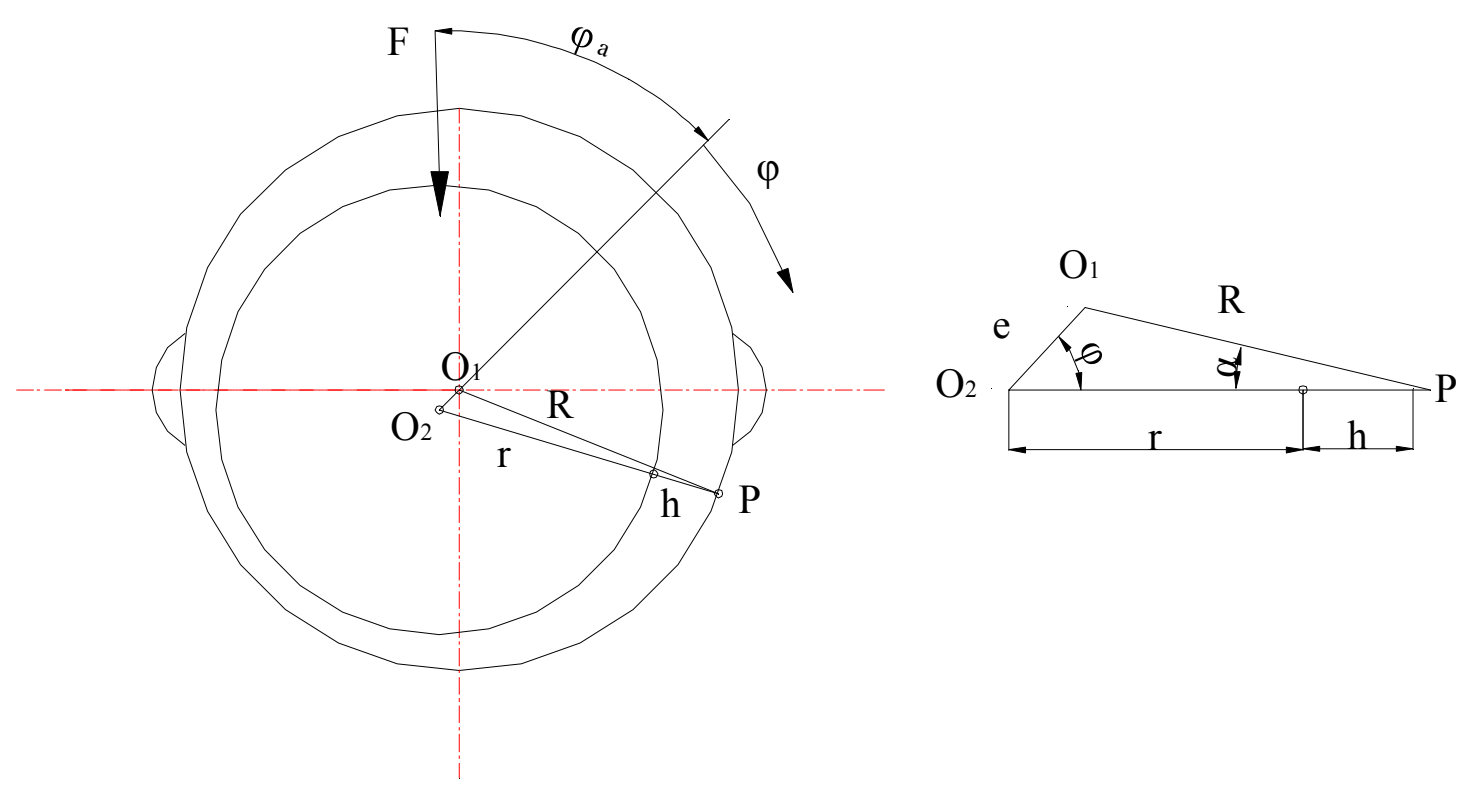

Fig. 1 The geometric parameters and oil pressure distribution of the crankshaft

In the Fig. $1, \mathrm{~h}$ is the oil film's thickness, $\mathrm{R}$ is radius of bearing, $\mathrm{r}$ is radius of main journal, $\mathrm{F}$ is the external load, $\varphi_{\mathrm{a}}$ is the angle of displacement, e is the eccentric distance.

Diametric clearance of plain journal bearing $\Delta=D-d$, radial clearance of plain journal bearing $\delta=R-r=\Delta / 2$, eccentricity $\chi=e / \delta$. In the $\Delta O_{1} O_{2} P$, with the cosine theorem

$$
\begin{gathered}
R^{2}=e^{2}+(r+h)^{2}-2 e(r+h) \cos \varphi \\
h=\delta(1+\chi \cos \varphi)
\end{gathered}
$$

Reynolds equation dimensionless simplified. Considering the needs of the stiffness and calculation scale, the paper calculate stiffness with the oil film pressure distribution and oil film thickness by a moment, the Reynolds equation changes to this

$$
\frac{\partial}{\partial x}\left(\frac{h^{3}}{\eta} \frac{\partial p}{\partial x}\right)+\frac{\partial}{\partial y}\left(\frac{h^{3}}{\eta} \frac{\partial p}{\partial y}\right)=6 U \frac{\partial h}{\partial x}
$$

In the coordinate axes, $\mathrm{X}$ is the tangential direction, $\mathrm{Y}$ is the radial direction. Tangential direction's dimensionless form: $x=R \varphi \quad, \quad 0 \leq \varphi \leq 2 \pi$; Radial direction's dimensionless form: $\lambda=2 y / L, L$ is the length of the main journal, $-1 \leq \lambda \leq 1$; dimensionless oil film stiffness: $H=h / \delta=1+\chi \cos \varphi$; the press's dimensionless form: $P=p \delta^{2} / 2 \eta U R$.

Take the above equations into Eq. 4, obtain dimensionless Reynolds equation:

$$
\frac{\partial}{\partial \varphi}\left(H^{3} \frac{\partial P}{\partial \varphi}\right)+\left(\frac{D}{L}\right)^{2} \frac{\partial}{\partial \lambda}\left(H^{3} \frac{\partial P}{\partial \lambda}\right)=3 \frac{\partial H}{\partial \varphi}
$$


In the Eq.5,D is the diameter of the crankshaft main journal .Then define $A_{i}=H^{3}, B_{i}=(D / L)^{2} H^{3}, C_{i}=-3 H^{2} \chi \sin \varphi, K_{i}=-3 \chi \sin \varphi$.Take them to the Eq.5 obtain the derivative of Eq. 5

$$
A_{i} \frac{\partial^{2} P}{\partial \varphi^{2}}+B_{i} \frac{\partial^{2} P}{\partial \lambda^{2}}+C_{i} \frac{\partial P}{\partial \varphi}=K_{i}
$$

Simplify Eq. 6, with the finite difference method:

$$
\left(\frac{\partial P}{\partial \varphi}\right)_{i, j}=\frac{P_{i+1, j}-P_{i-1, j}}{2 \Delta \varphi},\left(\frac{\partial^{2} P}{\partial \varphi^{2}}\right)_{i, j}=\frac{P_{i+1, j}-2 P_{i, j}+P_{i-1, j}}{\Delta \varphi},\left(\frac{\partial^{2} P}{\partial \lambda^{2}}\right)_{i, j}=\frac{P_{i, j+1}-2 P_{i, j}+P_{i, j-1}}{\Delta \lambda}
$$

We can get the final equation $(a=\Delta \varphi, b=\Delta \lambda)$ :

$$
\left(\frac{A_{i}}{a^{2}}+\frac{C_{i}}{2 a}\right) P_{i+1, j}+\frac{B_{i}}{b^{2}} P_{i, j+1}-2\left(\frac{A_{i}}{a^{2}}+\frac{B_{i}}{b^{2}}\right) P_{i, j}+\left(\frac{A_{i}}{a^{2}}-\frac{C_{i}}{2 a}\right) P_{i-1, j}+\frac{B_{i}}{b^{2}} P_{i, j-1}=K_{i}
$$

Take Eq. 7 into a simplified form

$$
E P_{j-1}+F P_{j}+E P_{j+1}=K_{j}
$$

\section{Solve the oil film stiffness of the HCPE crankshaft}

This part based on the HCPE crankshaft, give a MATLAB programming which apply successive over relaxation to get the oil film pressure load, then calculate the stiffness.

MATLAB program. The subroutine uses successive over relaxation that is an extension of Gauss-Seidel, a method to solve linear equation to realize the iteration. It is simple in form, easy to design.

Main parameters of the HCPE crankshaft: bearing radius: $R=30 \mathrm{~mm}$, crankshaft length: $L=30 \mathrm{~mm}$, contact angle: $\phi=120^{\circ}$, crankshaft rotational speed: $n=1000 \mathrm{r} / \mathrm{min}$, lubricate oil dynamic viscosity: $\eta=0.0215 \mathrm{~Pa} \cdot \mathrm{s}$, radial clearance of plain journal bearing: $\delta=0.015 \mathrm{~mm}$, initial oil pressure: $P_{0}=30 \mathrm{KPa}$.

Obtain the dimensionless oil film pressure $P$ is 0.819 form the consequence of MATLAB program, take it into $p=2 \eta U R P / \delta^{2}$ get the oil film pressure $p=33.9 \mathrm{MPa}$.

In the MATLAB computation results, get the maximum oil film thickness when the angle $\varphi=150^{\circ}, h_{\max }=\delta\left(1+\chi \cos 150^{\circ}\right)=7.2 \times 10^{-6} \mathrm{~m}$; obtain the minimum oil film thickness when the angle $\varphi=180^{\circ}, h_{\min }=\delta\left(1+\chi \cos 180^{\circ}\right)=6 \times 10^{-6} \mathrm{~m}$.

The calculation of oil film stiffness. Oil film stiffness is the derivative of oil film bearing capacity increment and film thickness increment

$$
K_{f}=\frac{\Delta W}{\Delta h}
$$

The increment of oil film bearing capacity 


$$
\Delta W=\iint P \mathrm{~d} x \mathrm{~d} y=\int_{-1}^{1} \int_{\varphi_{A}}^{\varphi_{B}} P R \mathrm{~d} \varphi \mathrm{d} \lambda=4.848 \times 10^{3} \mathrm{~N}
$$

In the Eq.10, $\varphi_{A}$ is the angle between Bearing arc starting point and the center of connecting and crankshaft, $\varphi_{B}$ is the angle between Bearing arc ending point and the center of connecting and crankshaft. The increment of film thickness $\Delta h=h_{\max }-h_{\min }=1.2 \times 10^{-6} \mathrm{~m}$.

$$
K_{f}=\frac{\Delta W}{\Delta h}=\frac{4.848 \times 10^{3} \mathrm{~N}}{1.2 \times 10^{-6} \mathrm{~m}}=4.04 \times 10^{9} \mathrm{~N} / \mathrm{m}
$$

\section{Conclusion}

The paper bases on the dimensionless Reynolds equation, refers to the Hydraulic Confined Piston Engine's (HCPE) crankshaft, writes the MATLAB programming, then calculate the oil film stiffness is $4.04 \times 10^{9} \mathrm{~N} / \mathrm{m}$.

\section{Acknowledgements}

The authors gratefully acknowledge the financial support from the National Natural Science Foundation (No.50975146) and Shandong Provincial Science Foundation of the P. R. of China (No. 2014ZRB01503) and outstanding graduate thesis Foundation of Qingdao University (No.YBPY2014012).

\section{References}

[1] Min Zheng, Wang Le, et al. Simulation on pressure distribution of sliding bearing based on MATLAB technology [J]. Lubrication engineering, 2008, 33(8): 51-53.

[2] Wen Shizhu, Huang Ping. Principles of tribology [M]. Beijing: Tsinghua University Press, 2008: 23-25.

[3] Pu Lianggui, Ji Minggang, et al. Design of machinery [M]. Beijing: Higher Education Press, 2006: 290-291.

[4] Ge Yuanshan, He Chao, et al. Experimental study on PAHs of diesel engine fuelled with biodiesel [J]. Transactions of CSICE, 2007, 25(2): 125-129.

[5] Zhang Hongxin, Zhang Tiezhu, Wang Weichao. Influence of valve' s characteristic on total performance of three cylinders internal combustion water pump [J]. Chinese Journal of Mechanical Engineering, 2009, 22(1): 91-96.

[6] Li Chun. Research on the stability of multi-leaf oil-foil bearing based on fitting nonlinear oil film force [D]. Harbin: Harbin Institute of Technology, 2013. 